\title{
Surface Micromachined Z-Axis Vibratory Rate Gyroscope
}

\author{
William A. Clark and Roger T. Howe \\ Berkeley Sensor \& Actuator Center \\ Deparment of Electrical Engineering and Computer Sciences \\ University of California at Berkeley \\ Berkeley, CA 94720-1770 \\ Roberto Horowitz \\ Department of Mechanical Engineering \\ University of California at Berkeley \\ Berkeley, CA 94720-1740
}

\begin{abstract}
We have designed and tested the first polysilicon surface micromachined vibratory rate gyroscope that is sensitive to angular rotation about an axis normal to the plane of the silicon chip, the Z-axis. The Analog Devices, Inc. BiMEMS technology was used to fabricate the gyroscope, which has as a demonstrated minimum detectable signal of $1 \mathrm{deg} / \mathrm{sec} / \mathrm{Hz}^{1 / 2}$ with performance projected to improve to $0.1 \mathrm{deg} / \mathrm{sec} / \mathrm{Hz}^{1 / 2}$ in a second-generation BiMEMS design.

Our design makes use of electrostatics to improve performance considerably. Electrostatic frequency tuning of the sense mode is used to enhance sensitivity. Quadrature error due to asymmetries in the vibrating structure is nulled by detecting the resulting motion and applying electrostatic forces by modifying voltages on the interdigitated sense comb fingers to cancel error precisely.
\end{abstract}

\section{INTRODUCTION}

Multi-axis sensors are highly desirable for inertial sensing of motion in three dimensions. Integration of three axis rotation rate sensors coupled with three axis accelerometers on a single chip would result in a monolithic, six degree-of-freedom inertial measurement system capable of measuring all possible translations and orientations. The gyroscope described here is sensitive to Z-axis angular rate and complements another polysilicon surface micro- machined rate gyroscope that is sensitive to rotations about the $X$ and $Y$ axes [1], thereby enabling single chip, three axis rate sensing.

The Z-axis gyroscope superficially resembles recently reported electrostatic comb-drive, lateral vibratory gyroscopes that sense rotations about an axis in the plane of the silicon chip[2][3][4][5]. In contrast to these other rate gyroscopes that exhibit out-of-plane deflections in response to an input Coriolis signal, the sense mode in the Z-axis gyroscope is an in-plane deflection that is orthogonal to the driven mode. Among the several benefits from this design approach are the use of differential interdigitated capacitors for both sense and force balance. As a result, a closed-loop Z-axis gyroscope can be realized in a single level of structural polysilicon.

\section{GYROSCOPE DESIGN}

\section{Generating and Sensing Coriolis Acceleration}

The operation of the Z-axis vibratory rate gyroscope is best illustrated by the spring-mass system shown in Fig. 1. As in all vibratory rate gyroscopes, a Coriolis acceleration is generated by a oscillating proof mass in a rotating reference frame. The proof mass is oscillated along the $\mathrm{X}$-axis (the driven mode), the reference frame rotates about the $Z$-axis, and the Coriolis acceleration is detected as deflections along the $Y$-axis (the sense mode).

The microelectromechanical sense element of the surface micromachined $\mathrm{Z}$-axis vibratory rate gyro is also shown in Fig. 1. The structure comprises three major elements: the suspension sys-

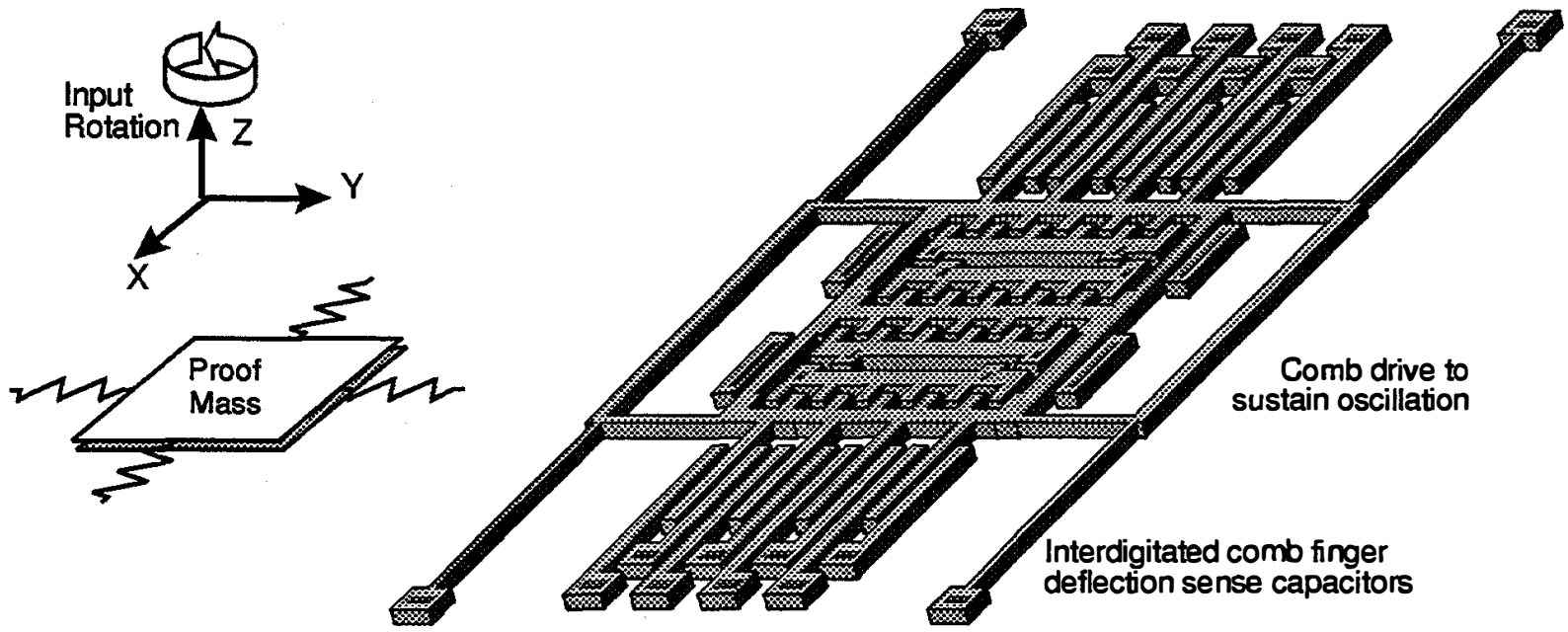

Figure 1. The spring-mass system at left, illustrates the basic operation of the Z-axis vibratory rate gyroscope. Coriolis acceleration results from the velocity of the oscillating proof mass and the rotation rate of the reference frame. The figure on the right represents the surface micromachined Z-axis vibratory rate gyro. The proof mass is made to vibrate along the X-axis using comb drives. Any deflections that result from Coriolis acceleration are detected in the sense mode using interdigitated comb fingers 
tem, the comb drive used to sustain oscillation[9], and the interdigitated comb fingers used both to detect deflections along the $\mathrm{Y}$-axis and to apply electrostatic force balance.

The oscillation of the gyroscope and the magnitude of the acceleration are given by Eq. 1 . As a point of reference, for a gyroscope with an oscillation amplitude, $X_{0}=1 \mu \mathrm{m}$, oscillation frequency, $\omega_{x}=20 \mathrm{kHz}$, and an input rotation rate, $\Omega_{z}=1 \mathrm{deg} / \mathrm{sec}$, the Coriolis acceleration has a magnitude of 0.45 milliG's.

$$
\begin{aligned}
x(t) & =X_{0} \sin \omega_{x} t \\
\ddot{y}_{\text {Coriolis }} & =2 \cdot \Omega_{z}(t) \times \dot{x}(t) \\
& =2 \cdot \Omega_{z}(t) \cdot X_{0} \cdot \omega_{x} \cdot \cos \omega_{x} t
\end{aligned}
$$

Notice that the Coriolis acceleration is an amplitude-modulated signal where the carrier frequency is the oscillation frequency and the rotation rate modulates the amplitude. The resulting Coriolis acceleration is a dual sideband signal centered on the oscillation frequency. Since the $\mathrm{Y}$-axis accelerations are proportional to velocity, the motion of the proof mass is elliptical as shown in Fig. 2.

\section{Mechanical Design Considerations}

The suspension is designed to be compliant along the $\mathrm{X}$ - and $\mathrm{Y}$-axes and as rigid as possible to other modes. In particular, the suspension must be rigid in rotation about the Z-axis since small rotations will degrade the performance of the gyroscope and large deflections result in comb fingers crashing. The H-suspension shown in provides the necessary translational compliance while rotational rigidity is provided by thickening two trusses as shown in Fig. 1.

\section{Quadrature Error}

Now consider one effect of manufacturing flaws. Suppose that the proof oscillates along an axis that is not exactly parallel to the $\mathrm{X}$-axis as shown in Fig. 2. This implies that there is some small fraction, $\varepsilon$, of the oscillation lies along the $Y$-axis. If we differentiate this displacement twice, we arrive at the acceleration due to offaxis oscillation as shown in Eq. 2. This acceleration term is referred to as quadrature error.

$$
\begin{aligned}
& y_{\text {Quadrature }}=-\varepsilon \cdot x(t) \\
& \ddot{y}_{\text {Quadrature }}=\varepsilon \cdot X_{0} \cdot \omega_{x}^{2} \cdot \sin \omega_{x} t
\end{aligned}
$$

Note the similarity between the quadrature error and the Coriolis acceleration: both are sinusoidal signals centered at the frequency of oscillation. However, they can be distinguished by their phase relative to the driven oscillation.

Quadrature errors can be quite large. The ratio of quadrature error and Coriolis acceleration is in Eq. 3. Using the previous example of an input rotation rate of $1 \mathrm{deg} / \mathrm{sec}$ and an oscillation frequency of $20 \mathrm{kHz}$, we find for the quadrature error to be as small as the Coriolis acceleration, the oscillation direction must be true to a factor of 1 part in 3.6 million. Due to manufacturing flaws and other imbalances[8], the quadrature error will be considerably larger than this and must be dealt with accordingly.

$$
\frac{\ddot{y}_{\text {Coriolis }}}{\ddot{y}_{\text {Quadrature }}}=\frac{2 \cdot \Omega_{z}(t) \cdot X_{0} \cdot \omega_{x}}{\varepsilon \cdot X_{0} \cdot \omega_{x}^{2}}=\frac{2}{\varepsilon} \frac{\Omega_{z}(t)}{\omega_{x}}
$$

\section{Electrostatic Quadrature Error Nulling}

Recall that quadrature error is a signal that is directly proportional to position of the proof mass. To cancel this error signal without effecting the Coriolis signal, we must apply a balancing force that is exactly proportional to position. This is achieved using the interdigitated position sense fingers. As the proof mass oscillates, the position sense capacitors change proportionately. A slight modification, $\Delta V$, of the $\mathrm{DC}$ bias voltage, $V_{D C}$, applied to the comb fingers as shown in Fig. 2 results in a net force directly proportional to position of the proof mass as given by Eq. 4 .

$$
\begin{aligned}
F_{y} & =\frac{C_{\text {overlap }}}{y_{0}} x(t)\left\{\left(V_{D C}+\Delta V\right)^{2}-\left(V_{D C}-\Delta V\right)^{2}\right\} \\
& =\frac{2 C_{\text {overlap }}}{y_{0}} V_{D C} \Delta V \cdot x(t)
\end{aligned}
$$
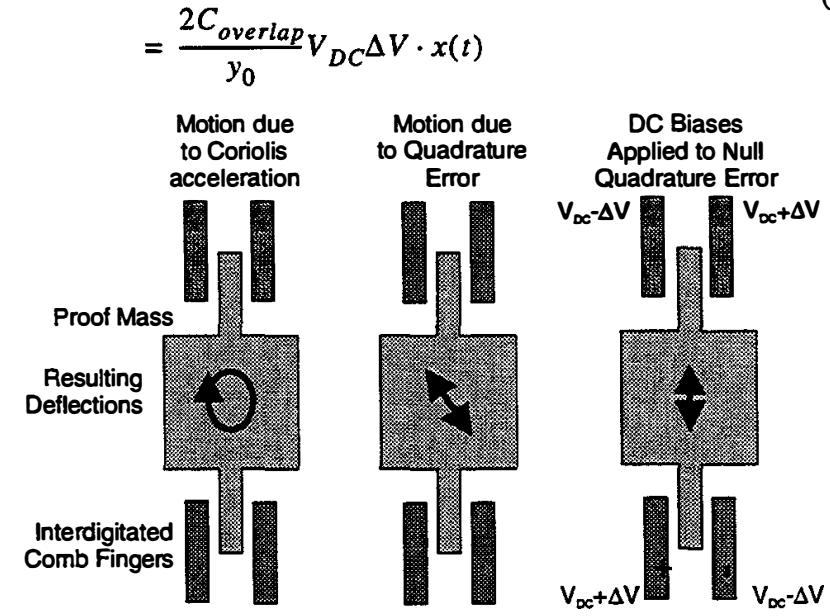

Figure 2. The motions caused by Coriolis acceleration and quadrature error are presented above. The right most figure shows our method used to null quadrature error. We use slight changes in the DC bias on the comb fingers to generate a force that is exactly proportional to position.

\section{Matching Modes for Improved Sensitivity}

Because the spring-mass system illustrated in Fig. 1 is a high $\mathrm{Q}$ second order system, the response of the proof mass to Coriolis acceleration can be enhanced. From Eq. 1, we know that Coriolis acceleration is a signal centered around the oscillation frequency. Therefore the relative values of the oscillation frequency and the $Y$-axis resonant frequency, $\omega_{y}$, have a dramatic effect on the response of the sense mode and hence the sensor sensitivity. If we match the Y-axis resonant frequency to the oscillation frequency, we can get a gain of $Q$ in the system response[6]. However, the bandwidth of the sensor is then limited to $\omega_{y} / \mathrm{Q}$. Given that system is run in a vacuum and $Q$ is typically greater than 10,000 , the system bandwidth for $\omega_{x}=\omega_{y}$ will be only a few Hertz.

For larger bandwidth and increased sensitivity, the Z-axis gyro is operated with a slight mismatch in resonant frequency[5]. The system response from Coriolis acceleration to $\mathrm{Y}$-axis displacement is given by Eq. 5 .

$$
\frac{y}{\Omega_{z}}=\frac{2 X_{0} \omega_{x} \sin \omega_{x} t}{\omega_{y}^{2}+\frac{j \omega_{x} \omega_{y}}{Q}-\omega_{x}^{2}} \approx \frac{X_{0}}{\omega_{x}} \frac{\omega_{x}}{\Delta \omega} \sin \omega_{x} t
$$

Provided that there is a means to tune the $\mathrm{Y}$-axis resonant frequency, it is desirable to operate the gyroscope with a $5-10 \%$ frequency mismatch, $\Delta \omega / \omega_{x}$, yielding a gain of 5-10 in sensitivity. 
Reducing the mismatch would increase the sensitivity but is probably difficult to maintain for any length of time.

Tuning of the $\mathrm{Y}$-axis resonant frequency to improve sensitivity is accomplished using electrostatic "negative" springs[6]. When a DC voltage is established between the moving structure and the comb fingers, an attractive force is applied to the structure. Using a first-order model for the parallel-plate capacitors, the resulting linearized spring force is given by Eq. 6 .

$$
\begin{aligned}
F_{y} & =\left\{\frac{1}{\left(y_{0}-y\right)^{2}}-\frac{1}{\left(y_{0}+y\right)^{2}}\right\} \frac{y_{0} C_{S} V_{D C}^{2}}{2} \\
k_{e} & \approx-\frac{2 C_{S}}{y_{0}} V_{D C}^{2} \\
\omega_{y} & =\sqrt{\frac{k_{y}+k_{e}}{M}}
\end{aligned}
$$

\section{Capacitive Deflection Sensing}

Interdigitated comb fingers, as shown in Fig. 1, are used to sense deflection. The comb fingers are arranged in a capacitor bridge, any deflection of the proof mass results in measurable changes the relative size of the capacitors.

There are two common amplifier configurations used for capacitive position sensing as shown in Fig. 3. The obvious difference between these two circuits is that the integrator offers the flexibility of trading bandwidth for sensitivity by varying the integrating capacitor size. The equivalent input referred noise (measured as a deflection of a sense capacitor plate) is the same for both configurations. However, the integrator offers the advantage of lower distortion. Some of the parasitic capacitors involved in MEMS are nonlinear which will vary the gain of a buffer and thereby result in distortion. Furthermore, the practice of bootstrapping parasitic capacitance is a form of positive feedback which exacerbates amplifier distortion. Because the integrator uses a fixed, linear capacitor, distortion is kept to a minimum.
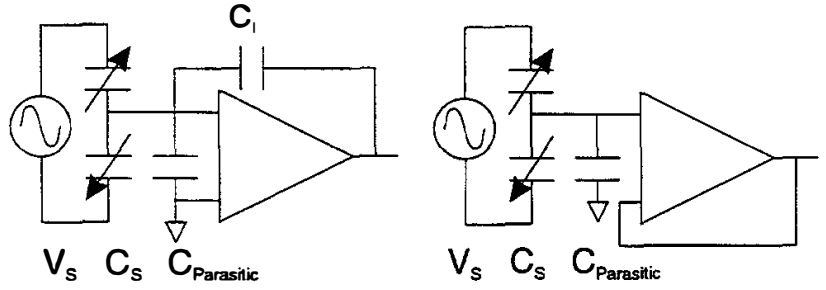

Figure 3. Capacitive position sensing using a voltage buffer and an integrator.

The integrator presents a difficult biasing problem. Ideally, biasing can be done by placing a very large resistor in parallel with the integrating capacitor as in Fig. 4. Any practical implementation of a large resistor results in considerable parasitic capacitance. A diode can also be used in parallel with the integrating capacitance, however, that adds a nonlinear capacitance to the integrating capacitance resulting in distortion. We have solved the biasing problem using subthreshold MOS devices. In subthreshold, the MOS devices exhibit extremely low transconductances and no source-todrain capacitance. The use of subthreshold MOS has resulted in a well behaved integrator operable down to $1 \mathrm{kHz}$ with a $50 \mathrm{~F}$ inte- grating capacitor without additional noise or distortion attributable to the bias circuitry.

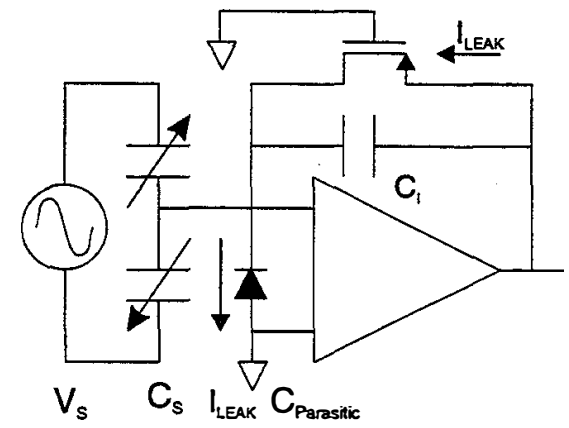

Figure 4. Integrator biasing using subthreshold MOS.

\section{Signal Processing}

At the core of the gyroscope signal conditioning is a phaselocked-loop (PLL) and several synchronous demodulators. The PLL is used to get robust, digital, signals that are in phase with the proof mass position and velocity. The phase accuracy of the PLL is critical to the operation of the gyroscope. Phase error will result in cross talk between Coriolis and quadrature signals. For the development in Eq. 7, we assume a phase error of $\theta_{n}(t)$.

Synchronous demodulators are used to extract the rotation rate information from the amplitude modulated Coriolis acceleration signal while rejecting the quadrature error. Signals from the PLL are mixed with measurements of the proof mass displacement as shown in Fig. 5 and Eq. 7.

$$
\begin{aligned}
y(t) & =\left[\frac{X_{0}}{\omega_{x}} \frac{\omega_{x}}{\Delta \omega} \Omega \cos (\omega t)+Y_{\text {Quadrature }} \sin (\omega t)\right] \\
\Omega_{\text {est }} & =2 \frac{\omega_{x}}{X_{0}} \frac{\Delta \omega}{\omega_{x}} y(t) \cos \left(\omega_{x} t+\theta_{n}(t)\right) \\
& =\Omega+\frac{\omega_{x} \Delta \omega_{X_{0}}}{\omega_{x}} Y_{\text {Quadrature }} \theta_{n}(t)+\text { High freq. terms }
\end{aligned}
$$

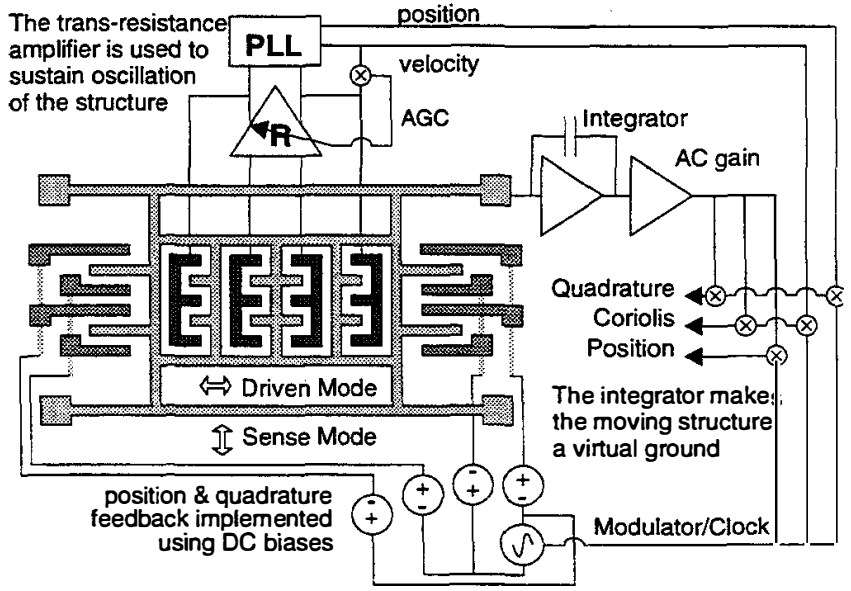

Figure 5. A block diagram of our Z-axis gyroscope and the associated signal processing elements. Shown above are the position sensing elements, the oscillation amp, and the demodulation required to operate the gyroscope.

\section{Noise Sources}

There are a number of possible noise sources. Principle among these are: Brownian noise, op-amp noise in the integrator, and PLL phase noise. Brownian noise represents the fundamental limit[10] 
and is given by Eq. 8. As an example, consider a gyroscope with mass, $M=0.2 \mu \mathrm{g}$, oscillation amplitude, $X_{0}=1 \mu \mathrm{m}$, a quality factor, $Q=10,000$, a bandwidth, $B W=100 \mathrm{~Hz}$, and resonant frequencies of $20 \mathrm{kHz}$. For this example, we find a Brownian noise floor, $\Omega_{n B}=0.06 \mathrm{deg} / \mathrm{sec}$. Because the gyroscope is run in vacuum and has a high $\mathrm{Q}$, Brownian noise is not the dominant noise source.

$$
\Omega_{n B}=\sqrt{\frac{k T \omega_{y}}{M Q \omega_{x}^{2}} \frac{B W}{x_{0}^{2}}}
$$

As seen in Eq. 7, phase noise in the PLL, represented by $\theta_{n}(t)$, can cause variable cross talk between quadrature and Coriolis signals. In our design, this effect is kept to a minimum due to the low phase noise in the PLL and nulled quadrature error.

The integrator op-amp noise is the dominant noise source. The input-referred noise of the op-amp noise is a function of the total capacitance attached to the summing node of the integrator, $C_{T}$, and is given by Eq. 9 . The electronic noise is at best $\Omega_{n E}=0.08 \mathrm{deg} / \mathrm{sec}$ for a modest CMOS process with $f_{T}=250 \mathrm{MHz}$, a frequency mismatch, $\Delta \omega=1 \mathrm{kHz}$, a sense voltage, $V_{S}=1$ volt, a sense capacitance, $C_{S}=100 \mathrm{fF}$, and the oscillation amplitude and finger spacing, $X_{0}=y_{0}=1 \mu \mathrm{m}$.

$$
\begin{array}{rlr}
\Omega_{n E} & =\frac{C_{T}}{C_{s}} \frac{y_{0}}{X_{0}} \frac{v_{n}}{V_{s}} \Delta \omega \sqrt{2 B W} \\
& =\frac{y_{0}}{X_{0}} \frac{\Delta \omega}{V_{s}} \sqrt{\frac{32}{3 \pi} \frac{k T}{C_{S}} \frac{B W}{f_{T}}} \quad \text { Optimal for } C_{\text {Parasitic }}=0
\end{array}
$$

\section{EXPERIMENTAL RESULTS}

Fig. 6 is a micrograph of the gyroscope sense element as implemented in the Analog Devices, Inc. BiMEMS process. Included on this die are the trans-resistance amplifier and the integrator. The remaining electronics was implemented off chip. The mechanical sensing element is about $1 \mathrm{~mm}$ across. A second, much improved, gyroscope has been implemented in the BiMEMS process.l

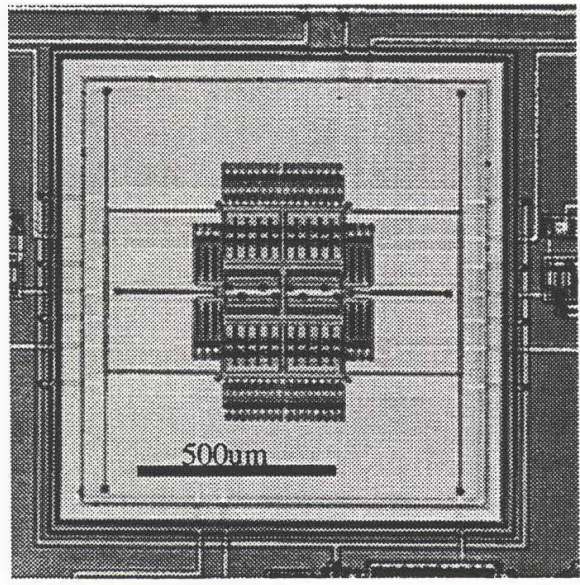

Figure 6. Die photo of Z-axis vibratory rate gyro. This structure makes extensive use of interdigitated comb fingers for both detection, excitation, and control.

\section{Resonant Frequency Tuning}

Fig. 7 plots the measured mechanical resonant frequencies as a function of the DC bias voltage applied to the position sense fingers. As expected, the Y-axis resonant frequency drops with increase bias voltage and the oscillation frequency of the proof mass remains constant at $12 \mathrm{kHz}$. Also shown are the resonant frequencies of the vertical and tipping modes that increase with DC bias. This increase is due to electrostatic levitation effects[11].

\section{Frequency Tuning}

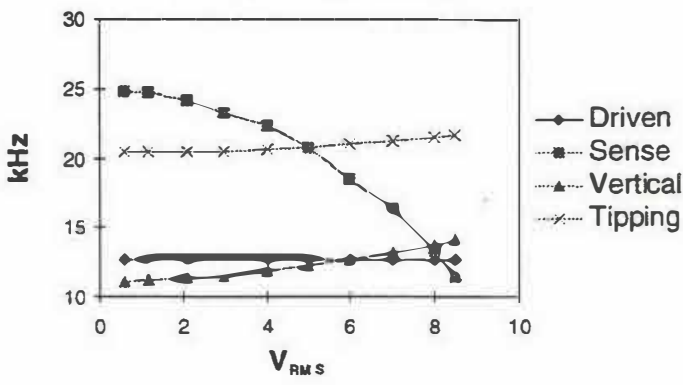

Figure 7. Frequency tuning as measured in the gyroscope. A DC bias combined with the modulation voltage establishes an RMS voltage between the comb fingers and the proof mass. The resulting electrostatic springs reduce the resonant frequency of the sense modes, raise the resonant frequencies of the out-of-plane modes, and leaves the driven mode unaffected.

\section{Quadrature Nulling}

This gyro has a means to null quadrature error. Measurements of the quadrature error and rotation rate signal in Fig. 8 demonstrate that the quadrature error signal can be controlled independently of the Coriolis signal.

\section{Quadrature Nulling}

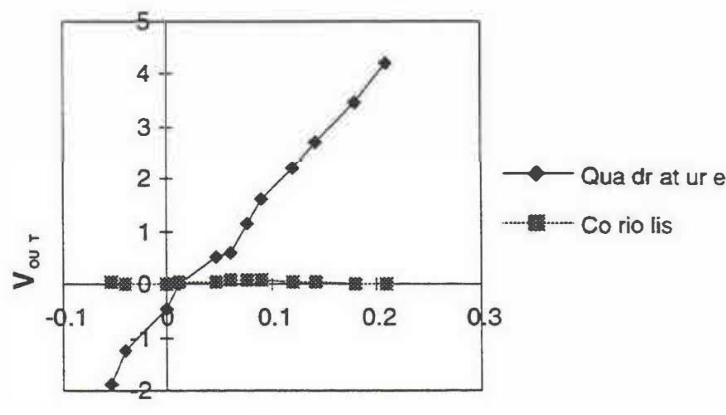

$\Delta \mathbf{V}$

Figure 8. This figure demonstrates the effect of the quadrature control scheme shown in Fig. 2 on measured rotation rate. Both the guadrature and rotation rate signals are plotted for a zero input rotation rate as the quadrature error control level is adjusted. 


\section{Rotation Rate Sensing}

An initial characterization of the gyroscope response is shown in Fig. 9, which is the measured output in response to a $1 \mathrm{~Hz}$, $5 \mathrm{deg} / \mathrm{sec}$ sine wave. The gyroscope is being operated with an oscillation frequency of $12 \mathrm{kHz}$ and a sense resonant frequency of about $12.5 \mathrm{kHz}$. The noise floor for this version of the Z-axis vibratory rate gyro is $1 \mathrm{deg} / \mathrm{sec} / \mathrm{Hz}^{1 / 2}$.

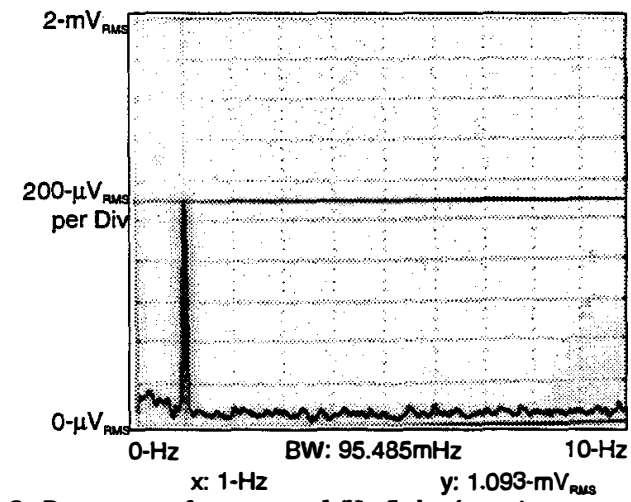

Figure 9. Response of gyro to a $1 \mathrm{~Hz} 5 \mathrm{deg} / \mathrm{sec}$ sine wave. Notice the low distortion and the noise floor that corresponds to $1 \mathrm{deg} / \mathrm{sec} / \mathrm{Hz}^{1 / 2}$.

\section{CONCLUSIONS}

We have designed, fabricated, and tested a polysilicon surfacemicromachined $\mathrm{Z}$-axis vibratory rate gyroscope that can be used in combination with other polysilicon surface inicromachined $\mathrm{X} / \mathrm{Y}$ gyroscopes to yield a 3 axis gyroscope on a single chip. An alternative approach to sensing $\mathrm{Z}$-axis angular rate is the resonant ring gyroscope[6]. Our design takes full advantage of an existing single structural layer MEMS process and the available electrostatic forces to yield a gyroscope with performance suitable for many real world applications.

Future research includes working toward more integrated versions of this gyroscope and working with others[1] to create a three axis gyroscope and a full six degree-of-freedom inertial navigation system.

\section{ACKNOWLEDGEMENTS}

This research was supported by the DARPA funded $i$ MEMS program, as a subconkact the Berkeley Sensor \& Actuator Center (BSAC) from Analog Devices, Inc. The authors thank Dr. Richard S. Payne and the Advanced Accelerometer Team for design support and for access to the BiMEMS technology. The Berkeley Sensor \&
Actuator Center is an NSF/Industry/University Cooperative Research Center.

\section{REFERENCES}

1. T. Juneau and A.P. Pisano, "Micromachined Dual Input Axis Angular Rate Sensor," Solid-State Sensor and Actuator Workshop, Hilton Head, SC, June 1996.

2. J. Bernstein et al, "A Micromachined Comb-Drive Tuning Fork Gyroscope," Digest IEEE/ASME Micro ElectroMechanical Systems (MEMS) Workshop, Ft. Lauderdale, FL, February 1993, pp 143-148.

3. P.B. Ljung, et al, "Micromachined Two Input Axis Angular Rate Sensor," ASME Dynamic Systems and Control Division, San Francisco, CA, November 1995, pp 957-962.

4. K. Tanaka et al, "A Micromachined Vibrating Gyroscope," Proceedings IEEE MicroElectroMechanical Systems 1995, Amsterdam, Netherlands, January 1995, pp 278-81.

5. J. Choi, et al, "Silicon Resonant Angular Rate Sensor by Reactive Ion Etching," Technical Digest of the 13th Sensor Symposium, 1995, pp 177-180.

6. M.W. Putty and Khalil Najafi, "A Micromachined Vibrating Ring Gyroscope," Solid-State Sensor and Actuator Workshop, Hilton Head, SC, June 1994, pp 213-220.

7. R.S. Payne, et al, "Surface Micromachining: From Vision to Reality," IEEE International Solid-State Circuits Conference, San Francisco, CA, February 1995, pp 164-165.

8. Howe, R.T., "Recent Advances in Surface Micromachining," Technical Digest of the 13th Sensor Symposium, Japan, 1995, pp. 1-8.

9. C.T.-C. Nguyen and R.T. Howe, "CMOS Micromechanical Resonator Oscillator" IEEE International Electronic Devices Meeting, Washington, D.C., December 1993, pp. 127-134.

10. T.B. Gabrielson, "Mechanical-Thermal Noise in Micromachined Acoustic and Vibration Sensors," IEEE Transactions on Electronic Devices, vol. 40, no. 5, May 1993, pp. 903-909.

11. W.C. Tang and R.T. Howe, "Electrostatic Comb Drive Levitation and Control Method,' LEEE/ASME Joumal of MicroElectroMechanical Systems, vol. 1, no. 4 December 1992, pp. 170178 , 